\title{
A NEW METHOD FOR QUANTIFICATION OF REGENERATED BONE TISSUE ON X-RAY IMAGES OF ELONGATED BONES
}

\author{
ANETA GĄDEK ${ }^{1}$, LESZEK WOJNAR ${ }^{1}$, MACIEJ TĘSIOROWSKI ${ }^{2}$ AND BARBARA JASIEWICZ ${ }^{2}$ \\ ${ }^{1}$ Institute of Computing Science, Krakow University of Technology, al. Jana Pawła II 37, 31-864 Krakow, \\ Poland, ${ }^{2}$ Jagiellonian University, University Orthopedics-Rehabilitation Hospital, ul. Balzera 15, 43-501 Zakopane \\ e-mails: 1wojnar@m7.pk.edu.pl; tesior@klinika.net.pl \\ (Accepted November 6, 2003)
}

\begin{abstract}
A new method for quantification of bone regenerate on the basis of computer-aided analysis of digitized Xray images is presented and its applicability in bone lengthening using Ilizarov method is demonstrated. In contrary to classical methods the internal part of the bone image is taken into consideration instead of the bone edges. Theoretical background of this concept is presented and experimentally verified. Experimental results show that the method proposed allows us for assessment of the bone regenerate, precise choice of the moment of external fixator removal as well as prediction of abnormalities in the osteogenesis process (excluding overall decalcification). However, the rules of interpretation of the results are not discussed in details.
\end{abstract}

Keywords: bone, image processing, osteogenesis, regenerate, X-ray.

\section{INTRODUCTION}

Computer-aided image analysis is widely used for medical purposes. Sophisticated monitoring and diagnostic systems using image data, neural networks or artificial intelligence are part of everyday practice in hospitals. Numerous problems are still far from a satisfactory solution because of the variety of diagnostic problems and the complexity of analyzed images. In this paper we present a new method for quantitative assessment of radiograms of limb elongated by Ilizarov method.

The disproportion of limbs is not only a cosmetic defect but can also lead to serious degenerative changes in hip joints of the healthy leg as well as to serious problems in everyday functioning of the patient. Satisfactory treatment is offered by distraction osteogenesis, one of the most popular limb lengthening methods, worked out by Ilizarov (Ilizarov 1989a,b; 1990). The first step in this process is putting on an external frame fixator. Next, the bone is cut and elongated by $1 \mathrm{~mm}$ per day in four steps $(0.25 \mathrm{~mm}$ per each step). Elongation is followed by stabilization that is approximately two times longer. Proper control of the development of a new bone structure (usually called regenerate) is decisive for the success of the whole treatment. The crucial point in this method is removal of the fixator at a proper time. If the fixator is removed too early, the regenerate may band or break. By contrast, too late a removal may lead to significant bone embrittlement.

$\mathrm{X}$-ray images are the basic source of information on the course of regenerate development during the stabilization period (Moosley, 1978; Hangartner, 1986; Huurman et al., 1987; Moreland et al., 1987; Peterson and Rohr, 1987, Walker et al., 1991; Noveline, 1999; Erbe and Ross, 2000). Traditional, visual and subjective assessment is still used to determine the time of fixator removal. Therefore, objective assessment of the X-ray images of the regenerate is very important for a surgeon.

Usually, it is postulated that the regenerate is well formed when its edges become continuous in X-ray images of the elongated bone (Minty et al., 1994; Maffuli et al., 1996). Unfortunately, even if we use the computer-aided methods for edge detection, the results are not univocal. This is shown in Fig. 1, which presents results of the bone edge detection based on three different filters: Sobel (Fig. 1b), morphological gradient (Fig. 1c) and morphological gradient applied to the initial image after histogram equalization (Fig. 1d). Clearly, different filters give completely different responses. For example, on the basis of Sobel filtration one can state that the bone edges are still invisible in the regenerate. By contrast, morphological filtering of the image after histogram equalization demonstrates continuous edges in the regenerated region. 
The above mentioned drawbacks are suppressed by a new method, proposed in this paper. This method allows for a more accurate assessment of the development in regenerate formation and, additionally, enables the surgeon to detect possible complications in the process earlier than it is possible using only the human visual system.

It should be stressed that the authors have not found in the literature any another quantitative and objective method for regenerate assessment.

\section{PROPOSED METHOD FOR REGENERATE QUANTIFICATION}

\section{General idea}

The method proposed allows for quantitative comparison of the regenerate and elongated bone. It is postulated that the osteogenesis process allows for removal of the fixator when the mineral density of the regenerate reaches the mineral density of the remaining part of the bone. It should be noticed, however, that the method presented here is insensitive to overall bone decalcification that may accompany the osteogenesis process.

In order to demonstrate the principle of this new method, two test images have been generated. The first test image depicts the bone structure reduced to a tube, that models the cortical bone as a part which absorbs the whole dose of X-ray radiation. The second test image depicts the structure of a bone as a homogeneous cylinder - in this case one assumes that all parts of the bone absorb a similar dose of radiation. Subsequently, the gray levels along an arbitrary chosen line (in test images and X-ray image of a bone) have been examined (Fig. 1). The analogy between gray levels' distribution of the bone reduced to a tube and real one is well visible in figs $2 \mathrm{a}$ and $3 \mathrm{c}$. Both of them have two extremes near the edges of analyzed objects.

This observation suggests that the bone structure is better approximated by a tube than by a cylinder. Therefore, its actual image mostly depends on its cortical parts, and the effect of the sponge bone is rather weak.

In order to verify the above described concept, the next two images were compared (Fig. 3a and 3b). They have been obtained under exactly the same conditions. The first image (Fig. 3a) presents the whole bone, whereas the second one (Fig. 3b) the same bone after mechanical removal of the sponge part. The differences between both images are practically insignificant, which is shown in the graph containing a comparison of the gray level distributions of both images (see Fig. 3c). The above described experiment validates the assumption that gray levels of the bone image are determined mainly by its cortical part.

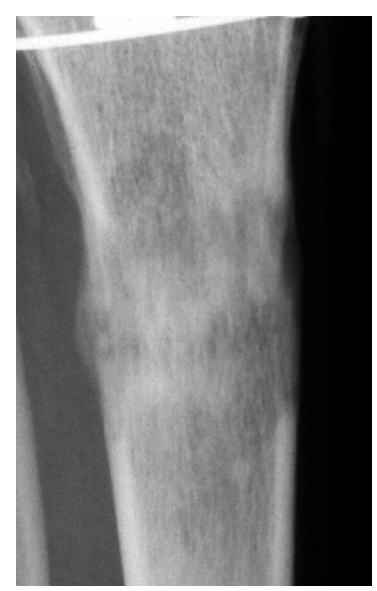

a) initial image of the bone with regenerate

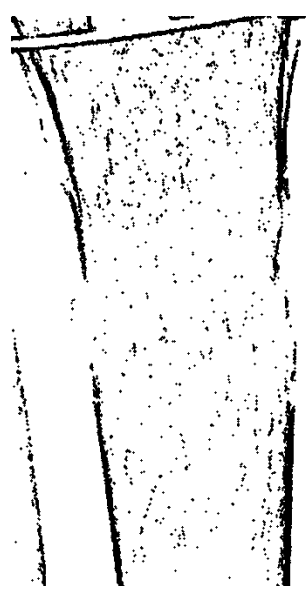

b) Sobel edges

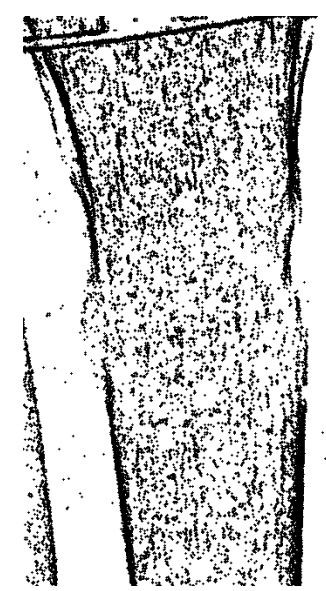

c) morphological gradient edges I

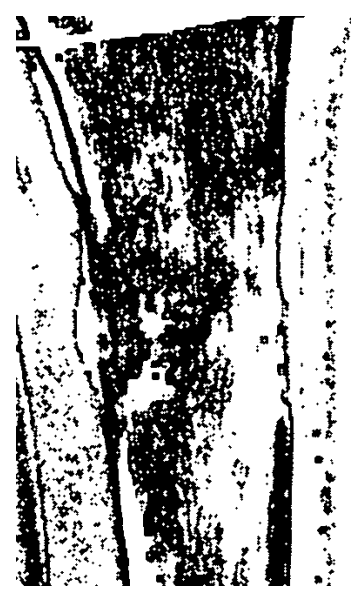

d) morphological gradient edges II

Fig. 1. The results of the bone edge detection by three different filters. 


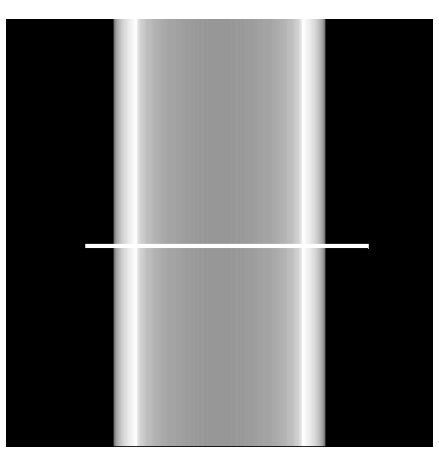

a) bone structure reduced to a tube

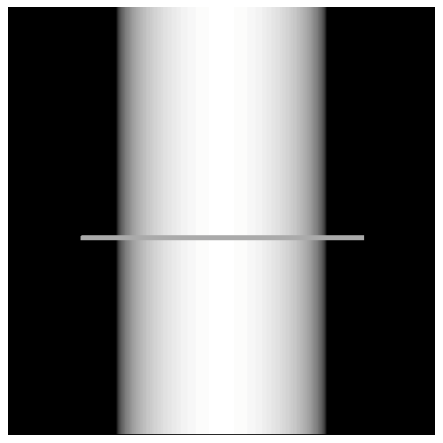

b) bone structure reduced to a cylinder

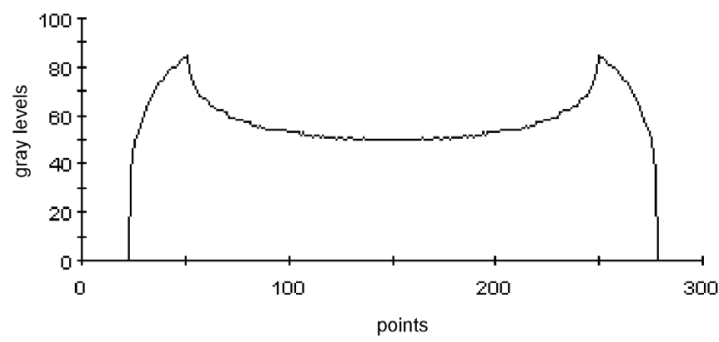

profile

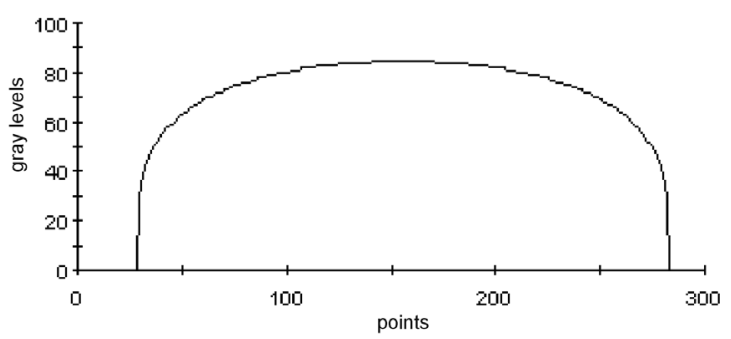

profile

Fig. 2. Gray level distribution of the test images presenting a tube a) and cylinder b).

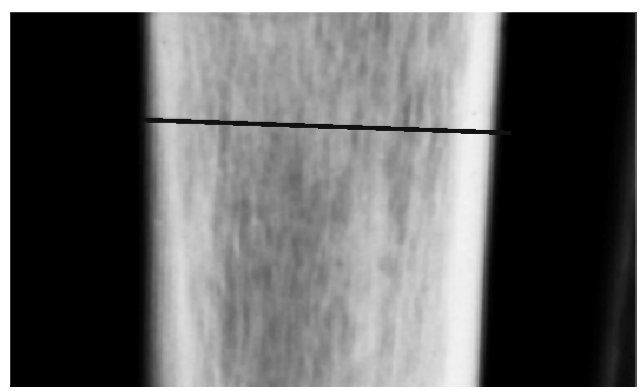

a) X-ray image of the bone with the sponge part and location of gray level profile

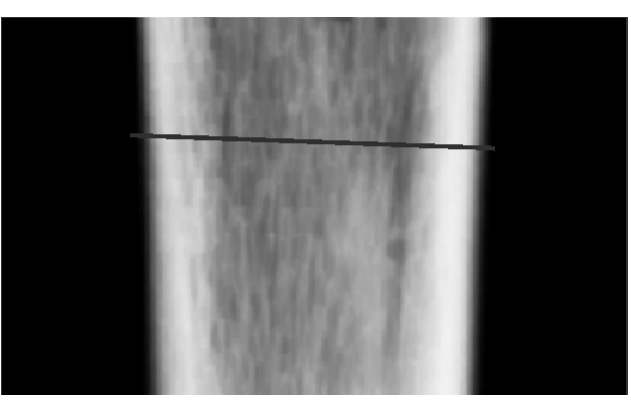

b) X-ray image of the bone with the sponge part mechanically removed and location of gray level profile

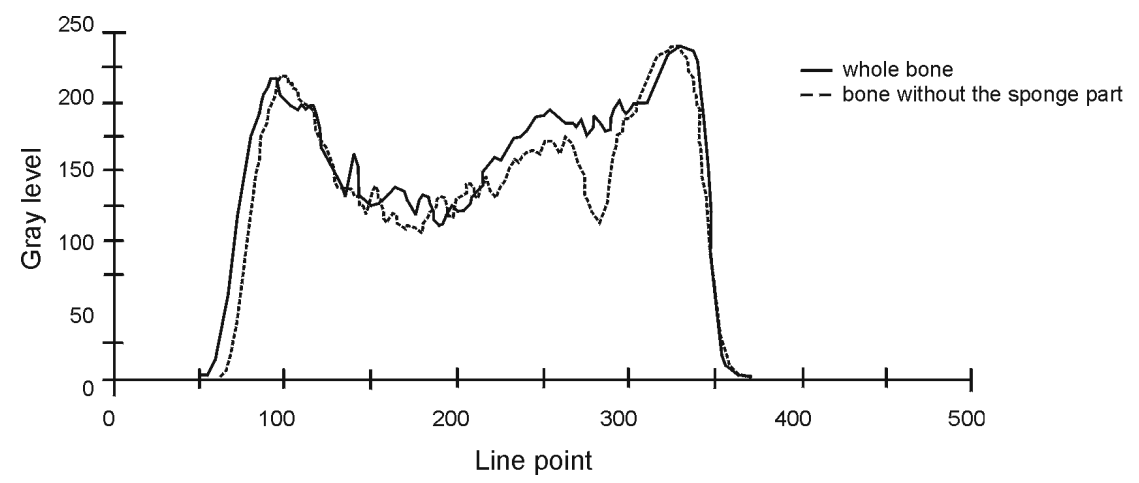

c) Comparison of X-ray level distribution

Fig. 3. Comparison of X-ray images and gray level distributions for the whole bone and the same bone after mechanical removal of the sponge part. 
As already mentioned, the traditional method of assessment of the regenerates maturity is based on the bone edge continuity (Minty et al., 1994; Maffuli et al., 1996). Such a method reflects our intuitive interpretation of X-ray images of the bone. It has to be noted that the X-ray image is a projection of a 3D object on a 2D surface and not a section of the analyzed object. If we assume that the bone structure can be reduced to a tube, as it is shown in Fig. 2a, its edges are the lightest areas because they absorb the highest dose of X-ray radiation (see Fig. 4).

The information obtained from the bone edges only cannot be treated as representative for the whole bone, as in this method only part of the bone is considered. Thus, it can be claimed that the traditional method is insufficient for reliable assessment of changes occurring during the stabilization process and the diagnosis based on this data may not be accurate enough. Additionally, as shown in Fig. 1, objective quantification of the bone edges was practically impossible. It is proposed in this work to take into consideration the internal part of the bone image as is determined predominantly by the cortical bone structure (see Fig. 5).

So, the essential novelty of this method of regenerate assessment lies in the fact that the middle part of the bone image instead of its edges is chosen for final analysis. In this way (Fig. 5), the analysis of the images of both projections (lateral and fore-back) yields information about changes in the whole distraction space. In other words, the whole volume of the regenerate is analyzed instead of its apparent edges.

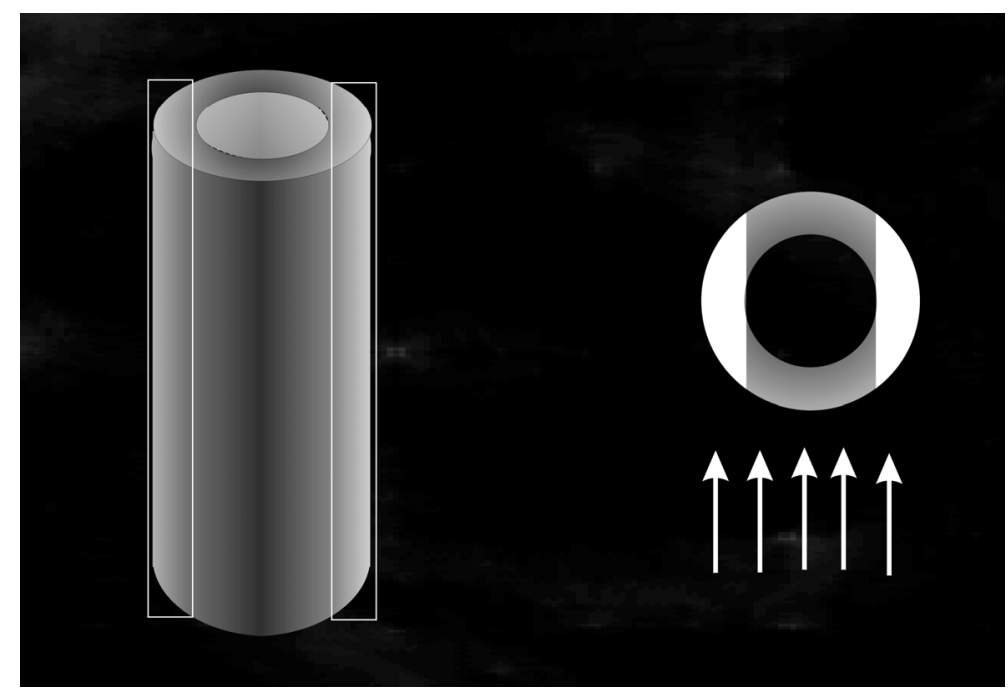

Fig 4. The bone edges constitute the lightest part of the object because mainly thicker layer of the tissues absorbs $X$-ray radiation.
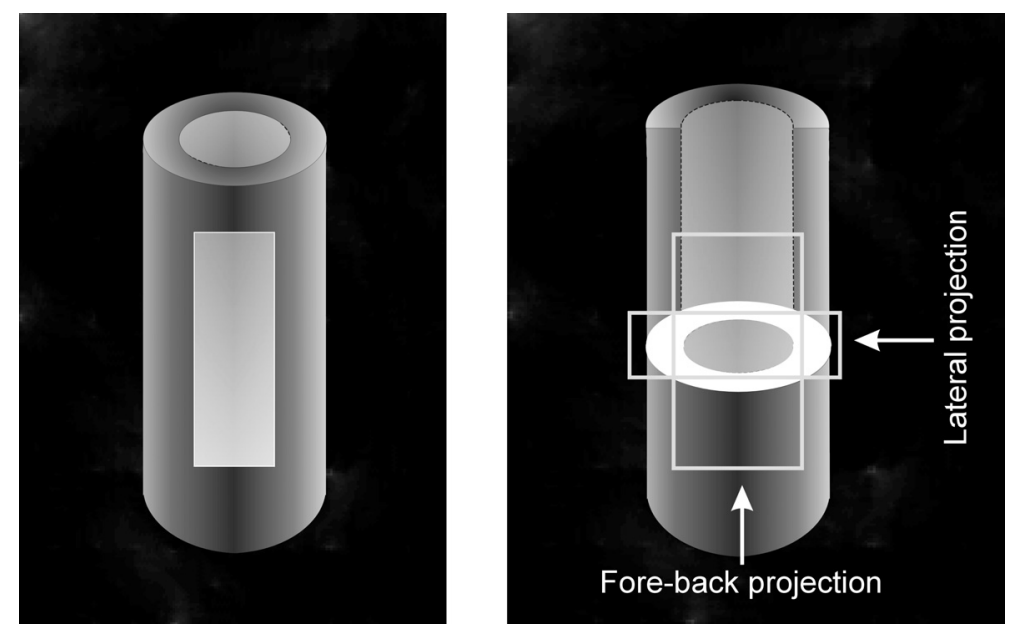

Fig. 5. The proposed method concentrates on the middle part of regenerate. The whole area of regenerate formation is considered (white area on the cross section). 


\section{ALGORITHM}

The main idea of the algorithm analyzing the regenerate is based on an experimental observation that the gray levels of the ideal bone structure change approximately in linear way along the bone axis (Fig. 6). Comparing (by dividing images) the real bone structure with a simulated one (generated by a computer) allows us to assess the extent of the regenerate development.

The simulated image of the bone is generated by the algorithm on the basis of pixel values representing the initial bone, lying outside the regenerate (Fig. 7). First, the image is median filtered in order to minimize the effect of possible artifacts. Next, the area of interest is chosen (in the current version of the method this step is performed in an interactive way) in such a way that its edges touch the bone-regenerate interface. New pixel values are computed on the basis pixels located at the safety distance form the area of interest (see Fig. 7 and 8a). This $2 \mathrm{~mm}$ distance has been experimentally determined and prevents the analysis of pixels belonging to the regenerate.

Let us denote the bone axis as $X$. In such a case pixels lying on o line parallel to this axis can be described as follows: the first pixel used for calculation has the abscissa equal to $x_{1}$ and the gray level value of $P_{1}$, whereas the second pixel the abscissa equal to $x_{2}$ and the gray level value of $P_{2}$. If $x_{2}>x_{1}$ than the pixel value $P_{s}$ located at $x$ in the simulated image (see Fig. $8 \mathrm{~b}$ ) can be computed as follows:

$$
P_{s}=\frac{x-x_{1}}{x_{2}-x_{1}} \cdot P_{1}+\frac{x_{2}-x}{x_{2}-x_{1}} \cdot P_{2}
$$

As the result of dividing the real and simulated images (see Fig. 8c) the coefficient of regenerate development $C_{R D}$ is obtained:

$$
C_{R D}=\frac{\sum_{i=1}^{n} \frac{P_{r}(i)}{P_{s}(i)}}{n}
$$

where: $C_{R D}$ - coefficient of regenerate development, $n$ - number of analyzed pixels in the area of interest, $P_{r}(i)$-value of the i-th pixel in the image of regenerate, $P_{s}(i)$ - value of the $\mathrm{i}$-th pixel in the simulated image.

Possibly the use of subtraction of the images instead of division for calculation of the $C_{R D}$ coefficient would be more intuitive. However, in the case of systematic changes in image contrast (being the consequence of different conditions of radiographic inspection) we could get different values of the coefficient. This would made comparison among different patients erroneous. The currently proposed formula is sensitive to changes in image brightness but this drawback can be relatively easily suppressed by normalization of the image.

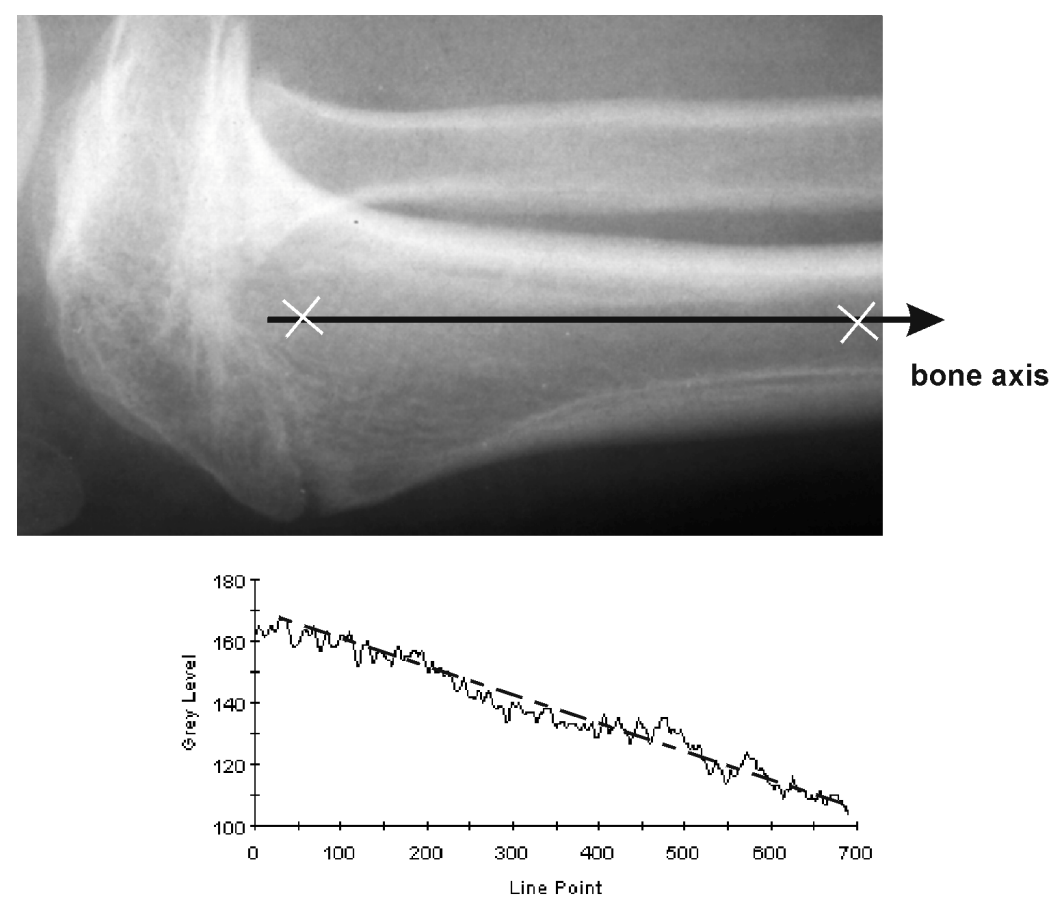

Fig. 6. The change in gray levels along bone axis may be approximated by linear function (dashed line on the chart). 


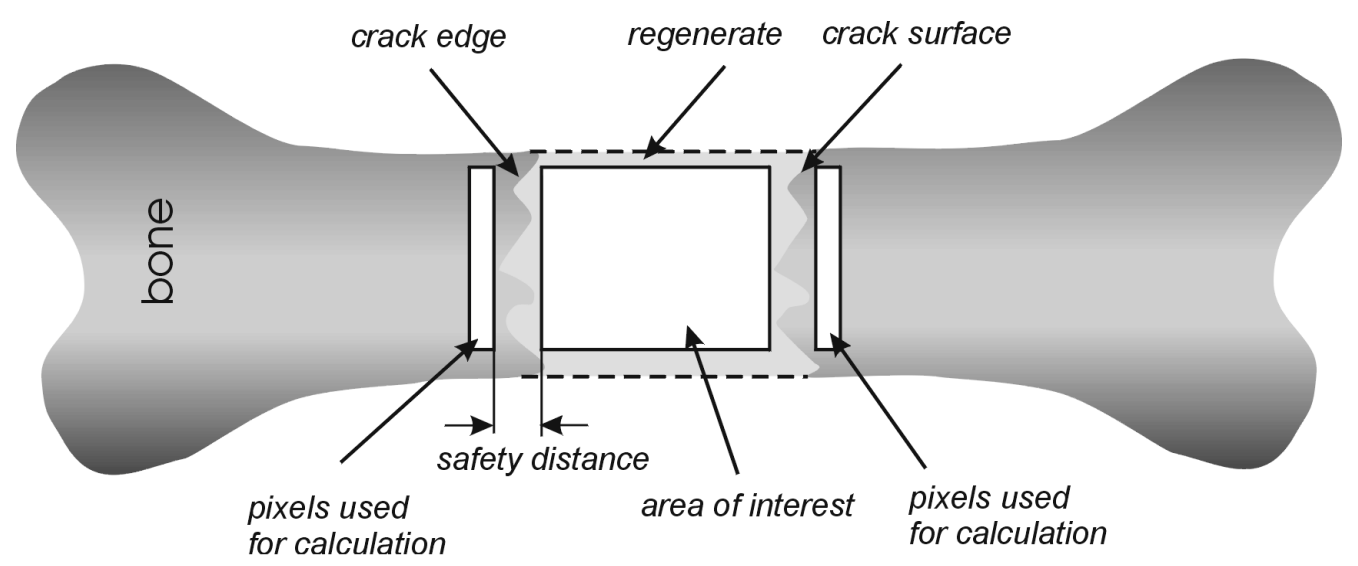

Fig. 7. Elongated bone, schematically. Details in the text.

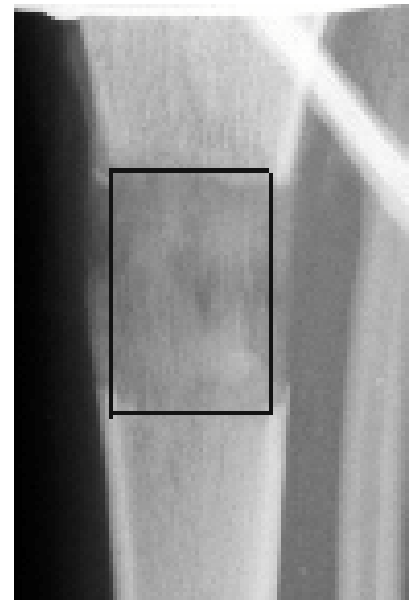

a) Bone at the beginning of stabilization with marked region of interest

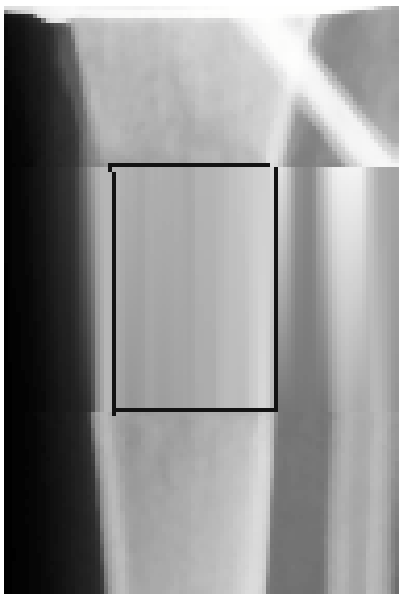

b) simulation of the model bone structure

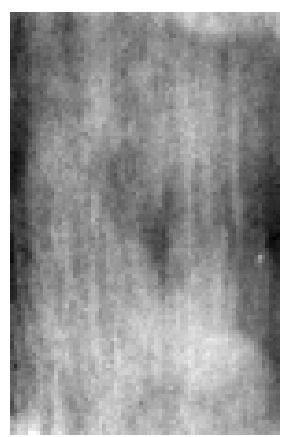

c) Final image - result of dividing the initial image and the model one

Fig. 8. Steps of the computer analysis of the regenerate at the beginning of stabilization.

\section{ILLUSTRATIVE RESULTS}

Numerous X-ray images illustrating the process of regenerate stabilization of bones lengthened by Ilizarov method were investigated. Only a small part of the results obtained will be presented below as the aim of this paper is to present the method itself and its applicability. Precise description of the rules of interpretation lies outside the scope of this work. Radiographs have been digitized using a scanner for transparent originals with 254 dpi resolution, thus giving $0.1 \mathrm{~mm}$ per pixel resolution of digital files. In order to get comparable results detailed guidelines for radiological examination have been worked out (Wojnar et al., 2002a,b). They strictly define radiological parameters, ray beam concentration spot and 
position of the wedge depending on the regenerate position. Such guidelines are necessary to standardize the results achieved in assessment of bone regenerate. In most cases X-ray images have been taken at fourweek internals.

The main result of the analysis of a single image is a value of $C_{R D}$ coefficient, which informs us about the degree of bone maturity. A $C_{R D}$ value close to 1 indicates that the calcification level is similar to that of the healthy bone. It is essential to remember that $C_{R D}$ is not sensitive to the process of overall decalcification, which may accompany the osteogenesis. This process can be detected using densitometric analysis, but usually can be neglected.

Currently the algorithm is semi-automatic (it is necessary to specify the test area prior to analysis) and therefore all the images were analyzed independently by a surgeon and a non-medical scientist in order to assess the effect of personal bias. The illustrative results of such simultaneous analysis are presented in Fig. 9. The observed deviations in results are the consequence of a purely independent (and, consequently, different) choice of the analyzed area. The scatter is not significant and the correlation between the results of two measurements is very high $(r=0.98)$.

In some cases the changes in regenerate, recorded in the images as darker or brighter areas, are invisible to a human eye (compare small images at the top of graph in Fig. 9). For example, the image corresponding to the 16-th week in Fig. 8 suggests significant drop in calcification, as the regenerate area is darker. In spite of this, the value of the coefficient of regenerate development is almost stable.

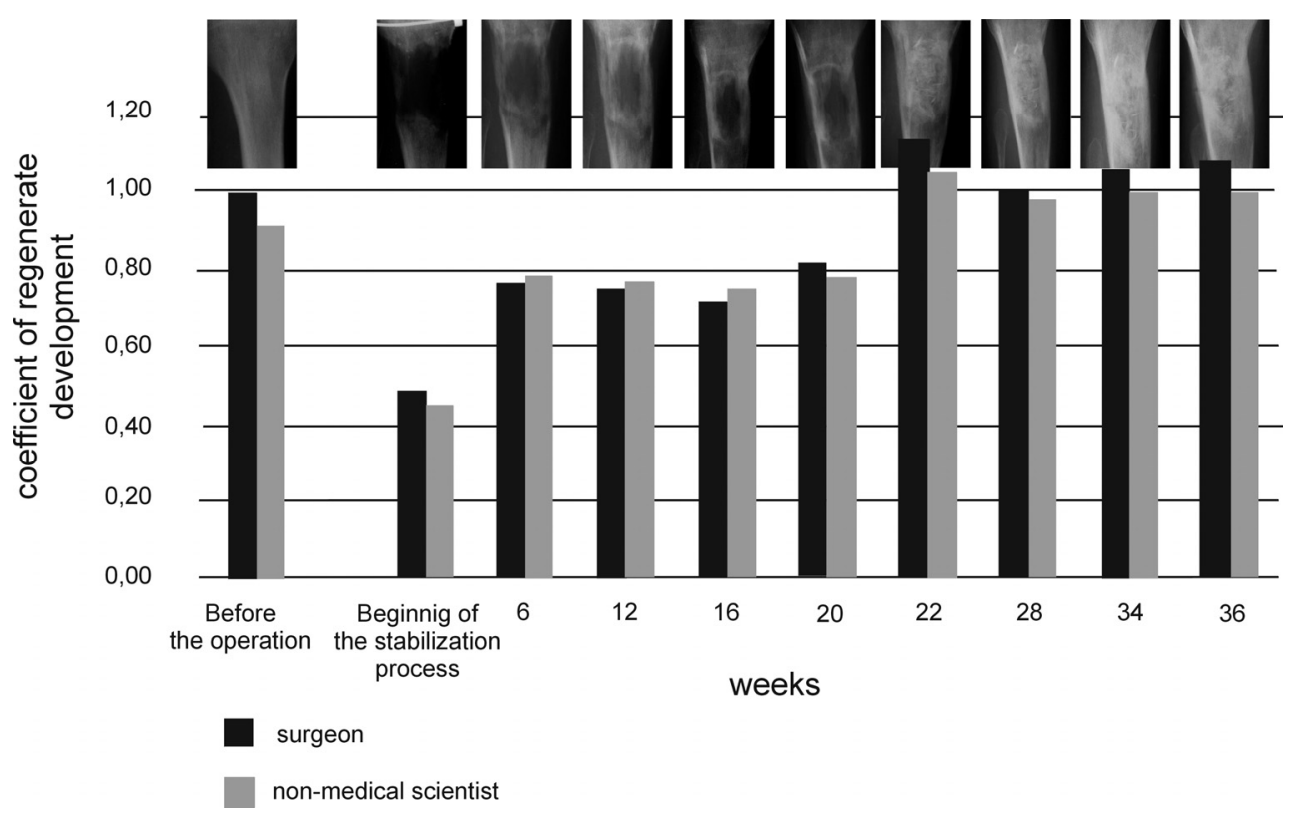

Fig. 9. Exemplary result of analysis for one patient. The fixator should be removed after 22 weeks.

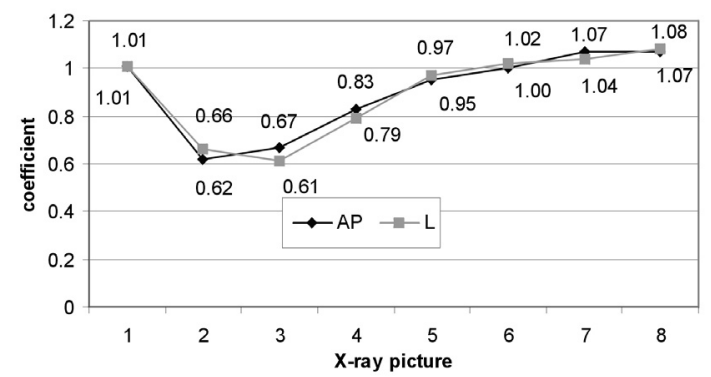

a) Mean coefficients' values for regenerate development during thigh lengthening

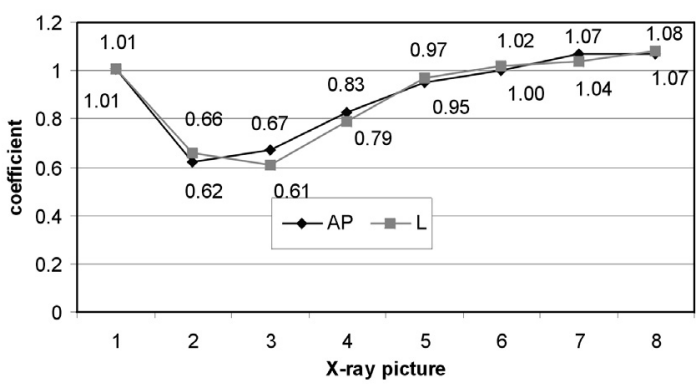

b) Mean coefficients' values for regenerate development during arm lengthening.

Fig. 10. Examples of proper stabilization in different bones (AP-X-ray images in the fore-back position, $L-X$-ray images in the lateral projection). 


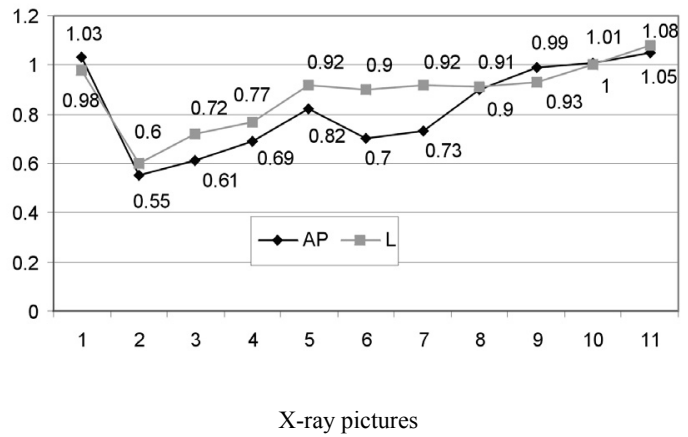

a) Regenerates' development coefficient value in case of too slow distraction osteognensis course (thigh lengthening).

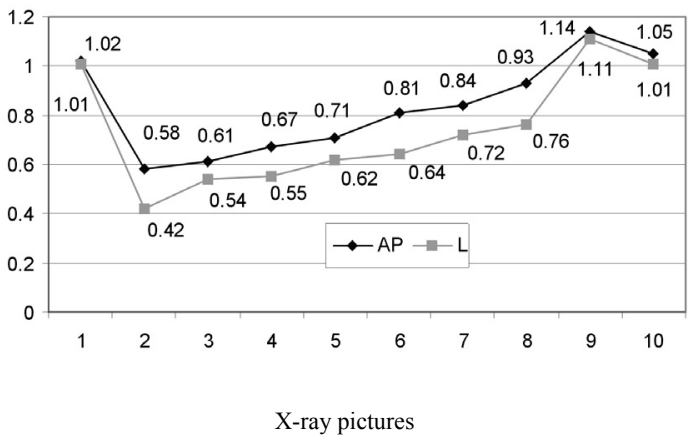

b) Regenerates' development coefficient value in the case of too slow distraction osteognensis course and cysts in the regenerate.

Fig. 11. Examples of stabilization with complications (AP - X-ray images in the fore-back position, $L-X$-ray images in the lateral projection).

The method described has been verified by clinical examination. Retrospective analysis of accessible medical data has shown a lack of complications in cases that have been independently assessed as successful by the computer method (compare Figs. 10 and 11). It has also been shown that real complication would have been detected earlier if the proposed computer-aided analysis was applied instead of traditional (visual or based on the edge detection) method. In the case of complications the coefficient of regenerate development takes values lower than the average for correct osteogenesis. However, formulation of appropriate standards requires more data than currently available. For example, there is a lack of standards for CT examination of the bone regenerate.

The research has been conducted in cooperation with a team of surgeons that are very experienced in lengthening bones using the Ilizarov method. As the risk of false diagnosis based on traditional method is higher in the case of less experienced teams, the proposed method may be particularly useful for them.

Presented results of analysis shown only usefulness of the proposed method. The way of the interpretation of obtained results and clinical verification overstep the bounds of this paper.

\section{CONCLUSIONS}

1. Objective, quantitative information about the degree of regenerate calcification obtained by computer-aided analysis is helpful in taking diagnostic decisions by a surgeon.

2. In contrast to traditional, visual assessment, computer aided image analysis of the regenerate image is more sensitive to subtle changes in the new bone structure that are invisible to a human eye. Consequently, this should be possible to detect significantly earlier any complications that appear in the process of stabilization.

3. Taking into account the internal part of the bone image simplifies quantification of the regenerate in comparison with the analysis of apparent bone edges.

\section{ACKNOWLEDGEMENTS}

Financial support from the State Committee for Scientific Research, grant No 7 T11E 03220 is gratefully acknowledged.

\section{REFERENCES}

Erbe MA, Ross PJ (2000).A technique for lower limb lengthening. Radiol Technol 71(5):497-9.

Hangartner TN (1986). The radiological measurement of bone. Can Assoc Radiol J 37:143-52.

Huurman WW, Stig FS, Anderson JC, Chu W (1987). Limb-Length discrepancy measured with computerized axial tomographic equipment. J Bone Joint Surg 67A(5):699-705.

Ilizarow GA (1989a). The tension-stress effect on the genesis and growth of tissues. Part 1 . The influence of stability of fixation and soft-tissue preservation. Clin Orthop 238:249-81.

Ilizarow GA (1989b). The tension-stress effect on the genesis and growth of tissues. Part 2. The influence of the rate and frequency of distraction. Clin Orthop 239:263-85.

Ilizarow GA (1990). Clinical application of tension stress effect for limb lengthening. Clin Orthop 250:8-26. 
Maffulli N, Lombari C, Matarazzo L, Nele U, Pagnona G, Fixsen JA (1996). A review of 240 patients undergoing distraction osteogenesis for congenital, posttraumatic or post-infective lower limb length discrepancy. J Am Coli Surg 182:394-402.

Minty I, Maffulli N, Hughes TH, Shaw DG, Fixsen JA (1994). Radiographic features of limb lengthening in children. A Radiol 35:555-9.

Moreland JB, Bassett LW, Hanker GJ (1987). Radiographic analysis of the axial alignment of the lower extremity. J Bone Joint Surg 69A(5):745-9.

Moosley CF (1978). A Straight Line Graph for Leg Length Discrepancies. Clin Orthop 136:33-40.

Novelline RA (1999). Squire's fundamentals of radiology. Harvard: University Press.
Paley D (1990). Problem, obstacles and complications of limb lengthening by Ilizarov technique. Clin Orthop 250:81-104.

Petersen TD, Rohr W (1987). Improved assesment of lower extremity alignment using new roentgenographic techniques. Clin Orthop 219:112-9.

Walker CW, Aronson J, Kaplan PA, Molpus WM, Seibert JJ (1991). Radiologic evaluation of limb lengthening procedures. AJR 156:353-8.

Wojnar L, Tęsiorowski M, Gądek A, Zarzycka M, Jasiewicz B, Kącki W (2002). Zastosowanie kliniczne ilościowej oceny regeneratu kostnego na zdjęciach rentgenowskich. Przegląd Lekarski 59(supl. 4):120-2.

Wojnar L, Kurzydłowski KJ, Szala J (2002). Praktyka analizy obrazu. Kraków: Polskie Towarzystwo Stereologiczne. 